\title{
A VOLTERRA-TYPE INTEGRATION OPERATOR ON FOCK SPACES
}

\author{
OLIVIA CONSTANTIN \\ (Communicated by Richard Rochberg)
}

AbStract. We study certain spectral properties and the invariant subspaces for some classes of integration operators of Volterra type on the Fock space.

\section{INTRODUCTION}

For analytic functions $f, g$ we consider the Volterra-type integration operator given by

$$
T_{g} f(z)=\int_{0}^{z} f(\zeta) g^{\prime}(\zeta) d \zeta
$$

The boundedness and compactness, as well as some spectral properties (such as Schatten class membership) of $T_{g}$ acting on various spaces of analytic functions of the unit disc $\mathbb{D}$ in $\mathbb{C}$ have been extensively investigated (see [2, 6] for Hardy spaces, [3, 5, 8, 17, 19] for weighted Bergman spaces, and [9, 10] for Dirichlet spaces, or the surveys [1, 20] and the references therein). Furthermore, the spectrum of $T_{g}$ on weighted Bergman spaces was recently characterized in [3].

In this paper we consider the operator $T_{g}$ acting on the Fock spaces $\mathcal{F}^{p}, p>0$ (here, the symbol $g$ is an entire function with $g(0)=0$ ). Recall that, for $p>0$, the Fock space $\mathcal{F}^{p}$ consists of entire functions $f$ for which

$$
\|f\|_{p}=\left(\frac{p}{2 \pi} \int_{\mathbb{C}}\left|f(z) e^{-\frac{|z|^{2}}{2}}\right|^{p} d A(z)\right)^{\frac{1}{p}}<\infty .
$$

The boundedness and compactness of $T_{g}$ follow by classical methods, and we include a sketch of their proofs in Section 3 for the sake of completeness. It turns out that, if $p \leq q, T_{g}: \mathcal{F}^{p} \rightarrow \mathcal{F}^{q}$ is bounded if and only if the symbol $g$ is a polynomial of degree $\leq 2$, while the necessary and sufficient condition for compactness is: $g$ is a polynomial of degree $\leq 1$. For $p>q$, the operator $T_{g}$ is bounded if and only if it is compact, and these are equivalent to the condition

$$
q>\frac{2 p}{p+2} \text { and } g(z)=a z \text { for some } a \in \mathbb{C} .
$$

In particular, this shows that the primitive of a function in $\mathcal{F}^{p}$ is in $\mathcal{F}^{q}$ for $q>$ $2 p /(p+2)$, and this result is sharp. In Section 4 we turn to the spectral properties of $T_{g}$. Regarding the Schatten class membership of $\left.T_{g}\right|_{\mathcal{F}^{2}}$, we show that, provided it is compact, $T_{g}$ fails to be Hilbert-Schmidt, but it belongs to all classes $\mathcal{S}^{p}$ for

Received by the editors May 30, 2011.

2010 Mathematics Subject Classification. Primary 30H20, 47B38.

Key words and phrases. Fock spaces, integration operator, spectrum, invariant subspaces. 
$p>2$. Moreover, we prove that the spectrum of $\left.T_{g}\right|_{\mathcal{F}^{2}}$ is a closed disc centred at the origin whose radius depends on the symbol $g$.

In the last section of this paper we characterize the invariant subspaces of the Volterra operator

$$
V f(z)=\int_{0}^{z} f(\zeta) d \zeta
$$

which is a particular case of the operator $T_{g}$, corresponding to $g(z)=z$. A complete description of the invariant subspaces of $V$, when acting on various classical spaces of analytic functions on the unit disc (Hardy spaces, weighted Bergman spaces, Dirichlet spaces) was obtained in [4. We prove the Fock space analogue of these results, showing that the proper invariant subspaces of $V: \mathcal{F}^{p} \rightarrow \mathcal{F}^{p}, p>0$, are given by

$$
A_{N}^{p}={\overline{\operatorname{Span}\left\{z^{k}: k \geq N+1\right\}}}^{\mathcal{F}^{p}},
$$

where $N$ is an arbitrary nonnegative integer.

\section{Preliminaries}

Recall that the point evaluation functionals are bounded on $\mathcal{F}^{p}, p>0$. More precisely, for every $f \in \mathcal{F}^{p}$, we have

$$
|f(z)| \leq e^{|z|^{2} / 2}\|f\|_{p}, \quad z \in \mathbb{C} .
$$

Moreover, the monomials $z^{n} / \sqrt{n !}, n \geq 0$, form an orthonormal basis for $\mathcal{F}^{2}$, and therefore the reproducing kernel of $\mathcal{F}^{2}$ is given by $K_{\lambda}(z)=e^{\bar{\lambda} z}$, i.e.

$$
f(\lambda)=\frac{1}{\pi} \int_{\mathbb{C}} f(z) e^{\lambda \bar{z}} e^{-|z|^{2}} d A(z), \quad \lambda \in \mathbb{C}, f \in \mathcal{F}^{2} .
$$

The following straightforward fact will be used in our further considerations: for an entire function $f$, the maximum principle and the subharmonicity of $|f|^{p}$ ensure that

$$
\begin{aligned}
\int_{\mathbb{C}} \frac{|f(z)|^{p}}{(1+|z|)^{p}} e^{-p|z|^{2} / 2} d A(z) & \sim \int_{|z|>1} \frac{|f(z)|^{p}}{(1+|z|)^{p}} e^{-p|z|^{2} / 2} d A(z) \\
& \sim \int_{|z|>1}\left|\frac{f(z)}{z}\right|^{p} e^{-p|z|^{2} / 2} d A(z) .
\end{aligned}
$$

Moreover, if $f(0)=0$, the above quantities are comparable to

$$
\int_{\mathbb{C}}\left|\frac{f(z)}{z}\right|^{p} e^{-p|z|^{2} / 2} d A(z) .
$$

The next proposition can be easily deduced from a result in [7]. We include a sketch of its proof for the sake of completeness.

Proposition 1. For an entire function $f: \mathbb{C} \rightarrow \mathbb{C}$ and $p>0$, the following holds:

$$
\int_{\mathbb{C}}|f(z)|^{p} e^{-p|z|^{2} / 2} d A(z) \sim|f(0)|^{p}+\int_{\mathbb{C}} \frac{\left|f^{\prime}(z)\right|^{p}}{(1+|z|)^{p}} e^{-p|z|^{2} / 2} d A(z) .
$$

Proof. It is enough to prove the claim for entire functions $f$ with $f(0)=0$. Indeed, the result for a general entire function $f$ follows by applying (3) to $z \mapsto f(z)-f(0)$. 
Suppose now that $f(z)=z g(z)$, where $g$ is entire. Then by Lemmas 5 -6 in [7] we have

$$
\int_{\mathbb{C}}|z g(z)|^{p} e^{-p|z|^{2} / 2} d A(z) \sim|g(0)|^{p}+\int_{\mathbb{C}}\left|g^{\prime}(z)\right|^{p} e^{-p|z|^{2} / 2} d A(z) .
$$

This is equivalent to

$$
\int_{\mathbb{C}}|f(z)|^{p} e^{-p|z|^{2} / 2} d A(z) \sim\left|f^{\prime}(0)\right|^{p}+\int_{\mathbb{C}}\left|\frac{z f^{\prime}(z)-f(z)}{z^{2}}\right|^{p} e^{-p|z|^{2} / 2} d A(z) .
$$

The integral on the right side is comparable to

$$
\int_{|z|>1}\left|\frac{z f^{\prime}(z)-f(z)}{z^{2}}\right|^{p} e^{-p|z|^{2} / 2} d A(z) \text {. }
$$

Using this fact, relation (2) and the obvious inequality $(a+b)^{p} \leq 2^{p}\left(|a|^{p}+|b|^{p}\right)$, we now obtain

$$
\int_{\mathbb{C}} \frac{\left|f^{\prime}(z)\right|^{p}}{(1+|z|)^{p}} e^{-p|z|^{2} / 2} d A(z) \lesssim \int_{\mathbb{C}}|f(z)|^{p} e^{-p|z|^{2} / 2} d A(z) .
$$

Hence one inequality in (3) is proven. We prove the reverse inequality by contradiction. Assume there exists a sequence of entire functions $f_{n}$ with $f_{n}(0)=0$ such that $\int_{\mathbb{C}}\left|f_{n}(z)\right|^{p} e^{-p|z|^{2} / 2} d A(z)=1$ and

$$
\int_{\mathbb{C}} \frac{\left|f_{n}^{\prime}(z)\right|^{p}}{(1+|z|)^{p}} e^{-p|z|^{2} / 2} d A(z) \leq \frac{1}{n}, \quad n \geq 1 .
$$

The expression $(1+|z|)$ is bounded above and below on any compact set in $\mathbb{C}$. Then by the subharmonicity of $\left|f^{\prime}(z)\right|^{p}$ and relation (5) we deduce that the sequence $f_{n}^{\prime}(z)$ converges to zero uniformly on the compact sets in $\mathbb{C}$ and, since $f_{n}(0)=0$, the same holds for $f_{n}$. We now write relation (4) for each $f_{n}$ and we use (2) to get

$$
\begin{aligned}
1 & \sim\left|f_{n}^{\prime}(0)\right|^{p}+\int_{\mathbb{C}}\left|\frac{z f_{n}^{\prime}(z)-f_{n}(z)}{z^{2}}\right|^{p} e^{-p|z|^{2} / 2} d A(z) \\
\lesssim & \left|f_{n}^{\prime}(0)\right|^{p}+\int_{|z|>1}\left|\frac{f_{n}^{\prime}(z)}{z}\right|^{p} e^{-p|z|^{2} / 2} d A(z)+\int_{|z|>1}\left|\frac{f_{n}(z)}{z^{2}}\right|^{p} e^{-p|z|^{2} / 2} d A(z) \\
\lesssim & \left|f_{n}^{\prime}(0)\right|^{p}+\frac{1}{n}+\int_{R>|z|>1}\left|\frac{f_{n}(z)}{z^{2}}\right|^{p} e^{-p|z|^{2} / 2} d A(z) \\
& +\frac{1}{R^{2}} \int_{|z|>R}\left|f_{n}(z)\right|^{p} e^{-p|z|^{2} / 2} d A(z) \\
\leq & \left|f_{n}^{\prime}(0)\right|^{p}+\frac{1}{n}+\int_{R>|z|>1}\left|\frac{f_{n}(z)}{z^{2}}\right|^{p} e^{-p|z|^{2} / 2} d A(z)+\frac{1}{R^{2}},
\end{aligned}
$$

for any $R>1$. Letting first $n \rightarrow \infty$ and then $R \rightarrow \infty$ above we obtain a contradiction. Thus (3) holds.

\section{BOUNDEDNESS AND COMPACTNESS}

The next theorem characterizes the boundedness and the compactness of $T_{g}$.

Theorem 1. (i) For $0<p \leq q$ the operator $T_{g}$ is bounded from $\mathcal{F}^{p}$ to $\mathcal{F}^{q}$ if and only if $g(z)=a z^{2}+b z$ for some $a, b \in \mathbb{C}$. Moreover, $T_{g}: \mathcal{F}^{p} \rightarrow \mathcal{F}^{q}$ is compact if and only if $g(z)=a z$ for some $a \in \mathbb{C}$. 
(ii) Assume $0<q<p$ and $g \not \equiv 0$. Then the operator $T_{g}$ is bounded from $\mathcal{F}^{p}$ to $\mathcal{F}^{q}$ if and only if $q>\frac{2 p}{p+2}$ and $g(z)=a z$, for some $a \in \mathbb{C} \backslash 0$. Furthermore, if $T_{g}: \mathcal{F}^{p} \rightarrow \mathcal{F}^{q}$ is bounded, then it is compact.

Proof. (i) We start by proving the sufficiency part of the statements in $(i)$. Assume $g(z)=a z^{2}+b z$. Then Proposition 1 gives

$$
\begin{aligned}
\left\|T_{g} f\right\|_{q}^{q} & \sim \int_{\mathbb{C}} \frac{\left|f g^{\prime}\right|^{q}}{(1+|z|)^{q}} e^{-q|z|^{2} / 2} d A(z) \\
& =\int_{\mathbb{C}}|f|^{q} \frac{|2 a z+b|^{q}}{(1+|z|)^{q}} e^{-q|z|^{2} / 2} d A(z) \\
& \lesssim\|f\|_{q}^{q} \leq\|f\|_{p}^{q},
\end{aligned}
$$

and the boundedness of $T_{g}$ follows from the inclusion $\mathcal{F}^{p} \subseteq \mathcal{F}^{q}$.

Let us now show that if $g^{\prime}$ is constant, then $T_{g}$ is compact. To this end, let $f_{n}$ be a sequence of entire functions such that $\sup _{n}\left\|f_{n}\right\|_{p}<\infty$ and $f_{n} \rightarrow 0$ uniformly on compact sets in $\mathbb{C}$. Also, let $R>0$. An application of Proposition 1 yields

$$
\begin{aligned}
\limsup _{n \rightarrow \infty}\left\|T_{g} f_{n}\right\|_{q}^{q} \sim & \limsup _{n \rightarrow \infty} \int_{\mathbb{C}}\left|f_{n}(z)\right|^{q} \frac{\left|g^{\prime}(z)\right|^{q}}{(1+|z|)^{q}} e^{-q|z|^{2} / 2} d A(z) \\
\sim & \limsup _{n \rightarrow \infty} \int_{|z| \leq R} \frac{\left|f_{n}(z)\right|^{q}}{(1+|z|)^{q}} e^{-q|z|^{2} / 2} d A(z) \\
& +\int_{|z|>R} \frac{\left|f_{n}(z)\right|^{q}}{(1+|z|)^{q}} e^{-q|z|^{2} / 2} d A(z) \\
\leq & \frac{1}{(1+R)^{q}} \limsup _{n \rightarrow \infty}\left\|f_{n}\right\|_{p}^{q} .
\end{aligned}
$$

Since $\sup _{n}\left\|f_{n}\right\|_{p}<\infty$, we now let $R \rightarrow \infty$ in the above relation to deduce that $T_{g} f_{n} \stackrel{\mathcal{F}^{q}}{\longrightarrow} 0$ as $n \rightarrow \infty$. Thus $T_{g}$ is compact.

Let us now prove the necessity. If $T_{g}$ is bounded, Proposition 1 yields

$$
\begin{aligned}
\left\|K_{\lambda}\right\|_{p}^{q} & \gtrsim\left\|T_{g} K_{\lambda}\right\|_{q}^{q} \sim \int_{\mathbb{C}} \frac{\left|g^{\prime}(z)\right|^{q}}{(1+|z|)^{q}}\left|K_{\lambda}\right|^{q} e^{-q|z|^{2} / 2} d A(z) \\
& \geq \int_{D(\lambda, 1)} \frac{\left|g^{\prime}(z)\right|^{q}}{(1+|z|)^{q}} e^{-q|z-\lambda|^{2} / 2} e^{q|\lambda|^{2} / 2} d A(z) \\
& \gtrsim e^{q|\lambda|^{2} / 2} \int_{D(\lambda, 1)} \frac{\left|g^{\prime}(z)\right|^{q}}{(1+|z|)^{q}} d A(z) \gtrsim e^{q|\lambda|^{2} / 2} \frac{\left|g^{\prime}(\lambda)\right|^{q}}{(1+|\lambda|)^{q}}, \quad \lambda \in \mathbb{C},
\end{aligned}
$$

by subharmonicity and the fact that $(1+|z|) \sim(1+|\lambda|)$ for $|z-\lambda|<1$. Since $\left\|K_{\lambda}\right\|_{p}=e^{|\lambda|^{2} / 2}$, the last relation above implies that

$$
\left|g^{\prime}(\lambda)\right| \lesssim 1+|\lambda|, \quad \lambda \in \mathbb{C},
$$

and hence $g$ is an analytic polynomial of degree $\leq 2$.

Now, if $T_{g}$ is compact, let $k_{\lambda}(z)=e^{-|\lambda|^{2} / 2} \bar{K}_{\lambda}(z)=e^{\bar{\lambda} z-|\lambda|^{2} / 2}$. Notice that $k_{\lambda} \rightarrow 0$ uniformly on compact sets as $|\lambda| \rightarrow \infty$. Moreover, $\left\|k_{\lambda}\right\|_{p}=1$. Hence $\left\|T_{g} k_{\lambda}\right\|_{q} \rightarrow 0$ as $|\lambda| \rightarrow \infty$. On the other hand, relation (77) shows that

$$
\left\|T_{g} k_{\lambda}\right\|_{q} \gtrsim \frac{\left|g^{\prime}(\lambda)\right|}{1+|\lambda|}, \quad \lambda \in \mathbb{C}
$$

which, in view of the previous considerations, forces $g^{\prime}$ to be constant. 
(ii) The proof of the necessity follows a similar approach to the one in [14]. Assume $T_{g}$ is bounded and let $\left(\lambda_{i}\right) \subset \mathbb{C}$ be a uniformly separated sequence, i.e. $\inf _{i \neq j}\left|\lambda_{i}-\lambda_{j}\right|>0$. Then, for any fixed radius $R>0$, there exists a positive integer $\mathcal{I}(R)>0$ such that any disc of radius $R$ contains at most $\mathcal{I}(R)$ points $\lambda_{i}$. We also assume that $\left(\lambda_{i}\right)$ is $\varepsilon$-dense for some $\varepsilon>0$, i.e. every point in $\mathbb{C}$ is within $\varepsilon$-distance of some $\lambda_{i}$. Then, for $\varepsilon$ sufficiently small (see Theorem 8.2 in [13] for $p \geq 1$ and Theorem 3.1 in 21] for $0<p<1$ ), it follows that for any sequence $\left(c_{i}\right) \in l^{p}$ the function defined by

$$
f(z)=\sum_{i \geq 1} c_{i} e^{\bar{\lambda}_{i} z-\left|\lambda_{i}\right|^{2} / 2}, \quad z \in \mathbb{C},
$$

belongs to $\mathcal{F}^{p}$ and $\|f\|_{p} \lesssim\left\|\left(c_{i}\right)\right\|_{l^{p}}$. By Khinchine's inequality we obtain

$$
\left(\sum_{i \geq 1}\left|c_{i}\right|^{2} e^{2 \operatorname{Re}\left(\bar{\lambda}_{i} z\right)-\left|\lambda_{i}\right|^{2}}\right)^{q / 2} \lesssim \int_{0}^{1}\left|\sum_{i \geq 1} r_{i}(t) c_{i} e^{\bar{\lambda}_{i} z-\left|\lambda_{i}\right|^{2} / 2}\right|^{q} d t
$$

where the $r_{i}$ are the Rademacher functions on $[0,1]$ (see [14]). Now denote

$$
d \mu(z):=\frac{\left|g^{\prime}(z)\right|^{q}}{(1+|z|)^{q}} e^{-q|z|^{2} / 2} d A(z)
$$

and use Fubini's theorem together with Proposition 1 to deduce

$$
\begin{aligned}
\int_{\mathbb{C}}\left(\sum_{i \geq 1}\left|c_{i}\right|^{2} e^{2 \operatorname{Re}\left(\bar{\lambda}_{i} z\right)-\left|\lambda_{i}\right|^{2}}\right)^{q / 2} d \mu(z) & \lesssim \int_{0}^{1} \int_{\mathbb{C}}\left|\sum_{i \geq 1} r_{i}(t) c_{i} e^{\bar{\lambda}_{i} z-\left|\lambda_{i}\right|^{2} / 2}\right|^{q} d \mu(z) d t \\
& \sim \int_{0}^{1}\left\|T_{g}\left(\sum_{i \geq 1} r_{i}(t) c_{i} e^{\bar{\lambda}_{i} z-\left|\lambda_{i}\right|^{2} / 2}\right)\right\|_{q}^{q} d t \\
& \leq \int_{0}^{1}\left\|T_{g}\right\|^{q}\left\|\sum_{i \geq 1} r_{i}(t) c_{i} e^{\bar{\lambda}_{i} z-\left|\lambda_{i}\right|^{2} / 2}\right\|_{p}^{q} d t \\
& \lesssim\left(\sum_{i \geq 1}\left|c_{i}\right|^{p}\right)^{q / p} .
\end{aligned}
$$

Since $\left(\lambda_{i}\right)$ is $\varepsilon$-dense, it follows that the discs $D\left(\lambda_{i}, 2 \varepsilon\right)$ cover $\mathbb{C}$, and therefore

$$
\begin{aligned}
\int_{\mathbb{C}}( & \left.\sum_{i \geq 1}\left|c_{i}\right|^{2} e^{2 \operatorname{Re}\left(\bar{\lambda}_{i} z\right)-\left|\lambda_{i}\right|^{2}}\right)^{q / 2} d \mu(z) \\
& \geq \mathcal{I}^{-1}(2 \varepsilon) \sum_{i \geq 1} \int_{D\left(\lambda_{i}, 2 \varepsilon\right)}\left|c_{i}\right|^{q} e^{q \operatorname{Re}\left(\bar{\lambda}_{i} z\right)-q\left|\lambda_{i}\right|^{2} / 2} d \mu(z) \\
& \gtrsim \sum_{i \geq 1}\left|c_{i}\right|^{q} \int_{D\left(\lambda_{i}, 2 \varepsilon\right)} \frac{\left|g^{\prime}(z)\right|^{q}}{(1+|z|)^{q}} d A(z) .
\end{aligned}
$$

Since $\left(c_{i}\right) \in l^{p}$ was arbitrarily chosen, by the previous relation together with (8) we get

$$
\left(\int_{D\left(\lambda_{i}, 2 \varepsilon\right)} \frac{\left|g^{\prime}(z)\right|^{q}}{(1+|z|)^{q}} d A(z)\right) \in l^{p /(p-q)} .
$$


By subharmonicity we have

$$
\frac{\left|g^{\prime}(z)\right|^{q}}{(1+|z|)^{q}} \lesssim \int_{D\left(\lambda_{i}, 2 \varepsilon\right)} \frac{\left|g^{\prime}(\zeta)\right|^{q}}{(1+|\zeta|)^{q}} d A(\zeta), \quad z \in D\left(\lambda_{i}, 3 \varepsilon / 2\right), i \geq 1,
$$

with constants depending on $\varepsilon$. We now raise both sides of the above inequality to the power $p /(p-q)$, then we integrate on $D\left(\lambda_{i}, 3 \varepsilon / 2\right)$ and sum over $i$ to get

$$
\begin{aligned}
\int_{\mathbb{C}}\left|\frac{g^{\prime}(z)}{(1+|z|)}\right|^{p q /(p-q)} d A(z) & \leq \sum_{i \geq 1} \int_{D\left(\lambda_{i}, 3 \varepsilon / 2\right)}\left|\frac{g^{\prime}(z)}{(1+|z|)}\right|^{p q /(p-q)} d A(z) \\
& \lesssim \sum_{i \geq 1}\left(\int_{D\left(\lambda_{i}, 2 \varepsilon\right)} \frac{\left|g^{\prime}(z)\right|^{q}}{(1+|z|)^{q}} d A(z)\right)^{p /(p-q)}<\infty .
\end{aligned}
$$

Thus $g^{\prime}$ is constant and $q>\frac{2 p}{p+2}$.

Now let us prove that $g^{\prime}$ constant and $q>2 p /(p+2)$ ensure the compactness of $T_{g}$. As before, let $f_{n}$ be a sequence of entire functions such that $\sup _{n}\left\|f_{n}\right\|_{p}<\infty$ and $f_{n} \rightarrow 0$ uniformly on compact sets in $\mathbb{C}$. Then, as in (6), for $R>0$, we have

$$
\begin{aligned}
\limsup _{n \rightarrow \infty}\left\|T_{g} f_{n}\right\|_{q}^{q} \sim & \limsup _{n \rightarrow \infty} \int_{|z| \leq R} \frac{\left|f_{n}(z)\right|^{q}}{(1+|z|)^{q}} e^{-q|z|^{2} / 2} d A(z) \\
& +\int_{|z|>R} \frac{\left|f_{n}(z)\right|^{q}}{(1+|z|)^{q}} e^{-q|z|^{2} / 2} d A(z) \\
\leq & \left(\int_{|z|>R} \frac{1}{(1+|z|)^{p q /(p-q)}} d A(z)\right)^{(p-q) / p} \limsup _{n \rightarrow \infty}\left\|f_{n}\right\|_{p}^{q}
\end{aligned}
$$

where the last step above follows by Hölder's inequality. As $\sup _{n}\left\|f_{n}\right\|_{p}<\infty$, we now let $R \rightarrow \infty$ in the above relation to deduce that $T_{g} f_{n} \stackrel{\mathcal{F}^{q}}{\longrightarrow} 0$ for $n \rightarrow \infty$. Thus $T_{g}$ is compact and the proof is complete.

\section{Schatten Class membership And the SpeCtrum of $T_{g}$ on $\mathcal{F}^{2}$}

Throughout this section, we shall consider the operator $T_{g}$ acting on $\mathcal{F}^{2}$. Recall that an operator $T$ acting on a Hilbert space belongs to the Schatten class $\mathcal{S}^{p}$, $p>0$, if the sequence of eigenvalues of $\left(T^{*} T\right)^{1 / 2}$ belongs to $l^{p}$.

Theorem 2. Suppose $T_{g}$ is compact. Then $T_{g}$ belongs to the Schatten class $\mathcal{S}^{p}$ for all $p>2$, but it fails to be Hilbert-Schmidt unless $g$ is constant.

Proof. By Theorem 1, the operator $T_{g}$ is compact if and only if $g^{\prime}$ is constant. Let us first show that $T_{g}$ fails to be Hilbert-Schmidt if $g^{\prime} \not \equiv 0$. Given an orthonormal basis $\left(e_{n}\right)$ in $\mathcal{F}^{2}$, we have $K_{z}(z)=e^{|z|^{2}}=\sum_{n \geq 1}\left|e_{n}(z)\right|^{2}$. We use this together with Proposition 1 to infer

$$
\sum_{n \geq 1}\left\|T_{g} e_{n}\right\|_{2}^{2} \sim \sum_{n \geq 1} \int_{\mathbb{C}} \frac{\left|e_{n}(z)\right|^{2}}{(1+|z|)^{2}} e^{-|z|^{2}} d A(z)=\int_{\mathbb{C}} \frac{K_{z}(z) e^{-|z|^{2}}}{(1+|z|)^{2}} d A(z)=\infty,
$$

which shows that $T_{g}$ is not Hilbert-Schmidt.

Given $p>2$, a bounded linear operator $A$ on a Hilbert space belongs to $\mathcal{S}^{p}$ if and only if $\sum_{n}\left\|A e_{n}\right\|^{p}<\infty$ for any orthonormal basis $\left(e_{n}\right)$ of the Hilbert space 
(see [11]). For an orthonormal basis $\left(e_{n}\right)$ of $\mathcal{F}^{2}$ we have, as before,

$$
\begin{aligned}
\sum_{n \geq 1}\left\|T_{g} e_{n}\right\|_{2}^{p} & \sim \sum_{n \geq 1}\left(\int_{\mathbb{C}} \frac{\left|e_{n}(z)\right|^{2}}{(1+|z|)^{2}} e^{-|z|^{2}} d A(z)\right)^{p / 2} \\
& \leq \sum_{n \geq 1}\left(\int_{\mathbb{C}}\left|e_{n}(z)\right|^{2} e^{-|z|^{2}} d A(z)\right)^{(p-2) / 2} \int_{\mathbb{C}} \frac{\left|e_{n}(z)\right|^{2} e^{-|z|^{2}}}{(1+|z|)^{p}} d A(z) \\
& =\sum_{n \geq 1} \int_{\mathbb{C}} \frac{\left|e_{n}(z)\right|^{2} e^{-|z|^{2}}}{(1+|z|)^{p}} d A(z)=\int_{\mathbb{C}} \frac{1}{(1+|z|)^{p}} d A(z)<\infty,
\end{aligned}
$$

by Hölder's inequality and since $\left(e_{n}\right)$ is an orthonormal basis. The proof is now complete.

Let us now turn to the spectrum of $T_{g}: \mathcal{F}^{2} \rightarrow \mathcal{F}^{2}$. We assume $T_{g}$ is bounded, and hence $g(z)=a z^{2}+b z$, for some $a, b \in \mathbb{C}$. Note first that for $\lambda \in \mathbb{C} \backslash\{0\}$, the equation

has the unique analytic solution

$$
f-\frac{1}{\lambda} T_{g} f=h
$$

$$
f(z)=R_{\lambda, g} h(z)=h(0) e^{g(z) / \lambda}+e^{g(z) / \lambda} \int_{0}^{z} e^{-g(\zeta) / \lambda} h^{\prime}(\zeta) d \zeta, \quad z \in \mathbb{C} .
$$

Thus the resolvent set of $T_{g}$ consists precisely of those points $\lambda \in \mathbb{C}$ for which $R_{\lambda, g}$ is a bounded operator on $\mathcal{F}^{2}$. Furthermore, the investigation of the spectrum of $T_{g}$ is related to the behavior of the exponentials $e^{g / \lambda}, \lambda \in \mathbb{C} \backslash\{0\}$, as was first pointed out by Pommerenke [18. Notice that for $h \equiv 1$ in the last relation above we get $R_{\lambda, g} 1=e^{g / \lambda}$. Hence $e^{g / \lambda} \in \mathcal{F}^{2}$ whenever $\lambda$ belongs to the resolvent set of $T_{g}$. This provides a sufficient condition for a point $\lambda$ to belong to the spectrum of $T_{g}$. A necessary and sufficient condition will be easily obtained once we have proved the next lemma.

Lemma 1. Assume $g(z)=a z^{2}+b z$ and $|\lambda|>2 a$. Then, for any entire function $f$, we have

$$
\int_{\mathbb{C}}\left|e^{g / \lambda} f\right|^{2} e^{-|z|^{2}} d A(z) \lesssim|f(0)|^{2}+\int_{\mathbb{C}} \frac{\left|e^{g / \lambda} f^{\prime}\right|^{2}}{(1+|z|)^{2}} e^{-|z|^{2}} d A(z) .
$$

Proof. Suppose first that $f(0)=f^{\prime}(0)=0$. A simple calculation based on the fact that the $\mathcal{F}^{2}$-norm of an entire function with Taylor series $\sum_{n \geq 0} a_{n} z^{n}$ is given by $\left(\sum_{n \geq 0}\left|a_{n}\right|^{2} n !\right)^{1 / 2}$ shows that

$$
\|f\|_{2} \leq\left\|\frac{f^{\prime}}{z}\right\|_{2}
$$

Replacing $f$ by $e^{g / \lambda} f$ above we obtain

$$
\left\|e^{g / \lambda} f\right\|_{2} \leq\left\|e^{g / \lambda}\left(\frac{2 a z+b}{\lambda z} f+\frac{f^{\prime}}{z}\right)\right\|_{2} \leq\left\|e^{g / \lambda} \frac{2 a z+b}{\lambda z} f\right\|_{2}+\left\|e^{g / \lambda} \frac{f^{\prime}}{z}\right\|_{2},
$$

and hence

$$
\left(1-\frac{|2 a|}{|\lambda|}\right)\left\|e^{g / \lambda} f\right\|_{2} \leq\left\|e^{g / \lambda} \frac{b f}{\lambda z}\right\|_{2}+\left\|e^{g / \lambda} \frac{f^{\prime}}{z}\right\|_{2}
$$

We claim that this implies that $\left\|e^{g / \lambda} f\right\|_{2} \lesssim\left\|e^{g / \lambda} f^{\prime} / z\right\|_{2}$. Indeed, if this did not hold, there would exist a sequence of entire functions $\left(f_{n}\right)$ with $f_{n}(0)=f_{n}^{\prime}(0)=0$ 
such that $\left\|e^{g / \lambda} f_{n}\right\|_{2}=1$ and $\left\|e^{g / \lambda} f_{n}^{\prime} / z\right\|_{2} \leq 1 / n$ for $n \geq 1$. The last inequality together with (1) show that the sequence $f_{n}^{\prime}(z)$ converges to zero uniformly on the compact sets in $\mathbb{C}$, and since $f_{n}(0)=0$, the same holds for $f_{n} / z$. On the other hand, for $R>0$, we deduce from (11) that

$$
\begin{aligned}
1-\frac{|2 a|}{|\lambda|} \leq & \left(\int_{|z| \leq R}\left|e^{g / \lambda} \frac{b f_{n}}{\lambda z}\right|^{2} e^{-|z|^{2}} d A(z)\right)^{1 / 2} \\
& +\frac{|b|}{|\lambda| R}\left(\int_{|z|>R}\left|e^{g / \lambda} f_{n}\right|^{2} e^{-|z|^{2}} d A(z)\right)^{1 / 2}+\frac{1}{n} \\
\leq & \left(\int_{|z| \leq R}\left|e^{g / \lambda} \frac{b f_{n}}{\lambda z}\right|^{2} e^{-|z|^{2}} d A(z)\right)^{1 / 2}+\frac{|b|}{|\lambda| R}+\frac{1}{n} .
\end{aligned}
$$

We first let $n \rightarrow \infty$, and subsequently $R \rightarrow \infty$, in the above relation to obtain a contradiction. Thus

$$
\left\|e^{g / \lambda} f\right\|_{2} \lesssim\left\|e^{g / \lambda} f^{\prime} / z\right\|_{2} \sim\left(\int_{\mathbb{C}} \frac{\left|e^{g / \lambda} f^{\prime}\right|^{2}}{\left(1+|z|^{2}\right)} e^{-|z|^{2}}\right)^{1 / 2}
$$

The desired result for a general $f$ can be easily deduced by applying the above inequality to $z \mapsto f(z)-f(0)-f^{\prime}(0) z$.

Theorem 3. Suppose $g(z)=a z^{2}+b z$ for some $a, b \in \mathbb{C}$. Then the spectrum of $T_{g}$ is the closed disc centred at the origin and of radius $2|a|$.

Proof. Let $\rho\left(T_{g}\right)$ denote the resolvent set of $T_{g}$. As mentioned before, if $\lambda \in \rho\left(T_{g}\right)$, then $e^{g / \lambda} \in \mathcal{F}^{2}$. A straightforward calculation using polar coordinates shows that, if $|\lambda|<2|a|$, then $e^{g / \lambda} \notin \mathcal{F}^{2}$, and hence $\overline{D(0,2|a|)}$ is contained in the spectrum of $T_{g}$. Let us now prove that if $|\lambda|>2|a|$, the formal resolvent $R_{\lambda, g}$, given by (10), is a bounded operator. If $|\lambda|>2|a|$, then clearly $e^{g / \lambda} \in \mathcal{F}^{2}$. We first apply Lemma 1. and subsequently Proposition 1, to deduce

$$
\begin{aligned}
\left\|R_{\lambda, g} f\right\|_{2} & \leq|f(0)|\left\|e^{g(z) / \lambda}\right\|_{2}+\left\|e^{g(z) / \lambda} \int_{0}^{z} e^{-g(\zeta) / \lambda} f^{\prime}(\zeta) d \zeta\right\|_{2} \\
& \lesssim\|f\|_{2}+\left(\int_{\mathbb{C}} \frac{\left|f^{\prime}(z)\right|^{2}}{(1+|z|)^{2}} e^{-|z|^{2}} d A(z)\right)^{1 / 2} \lesssim\|f\|_{2},
\end{aligned}
$$

which shows that $R_{\lambda, g}$ is bounded. Thus $\rho\left(T_{g}\right)=\{\lambda \in \mathbb{C}:|\lambda|>2|a|\}$.

\section{INVARIANT SUBSPACES OF THE VOLTERRA OPERATOR}

In the particular case $g(z)=z$, the operator $T_{g}$ becomes the Volterra operator

$$
V f(z)=\int_{0}^{z} f(\zeta) d \zeta, \quad z \in \mathbb{C}, \quad f \in \mathcal{F}^{p}, p>0 .
$$

By Theorem 1, the operator $V: \mathcal{F}^{p} \rightarrow \mathcal{F}^{p}$ is compact. The aim of this section is to characterize the invariant subspaces of $V$. A closed subspace $\mathcal{M}$ in $\mathcal{F}^{p}$ is called invariant for $V$ if $V \mathcal{M} \subset \mathcal{M}$. We start with the following simple observation.

Remark 1. The spectrum of the Volterra operator is the set $\{0\}$.

Proof. Notice that $V$ has no eigenvalues, since for any $f \in \mathcal{F}^{p} \backslash\{0\}$ the function $V f$ has a zero at $z=0$ of one order greater than the corresponding order for $f$. Since $V$ is compact and has no eigenvalues, its spectrum must be $\{0\}$. 
Remark 2. For $x_{0}=p>0$ we consider the sequence defined inductively by

$$
x_{n+1}=\frac{2 x_{n}}{x_{n}+2}, \quad n \geq 0 .
$$

Note that $\left(x_{n}\right)$ is decreasing and $\lim _{n \rightarrow \infty} x_{n}=0$. Then, for any nonnegative integer $M$, it follows by Theorem 1 (ii) that the operator $V^{M}$ is bounded from $\mathcal{F}^{p}$ to $\mathcal{F}^{q}$ if $q>x_{M}$.

Theorem 4. Assume $p>0$. Then $\mathcal{M} \subset \mathcal{F}^{p}$ is a proper invariant subspace of $V: \mathcal{F}^{p} \rightarrow \mathcal{F}^{p}$ if and only if there exists a nonnegative integer $N$ such that

$$
\mathcal{M}=\left\{f \in \mathcal{F}^{p}: f^{(k)}(0)=0 \text { for } 0 \leq k \leq N\right\}={\overline{\operatorname{Span}\left\{z^{k}: k \geq N+1\right\}}}^{\mathcal{F}^{p}} .
$$

Proof. Clearly, the sets

$$
A_{N}^{p}:=\left\{f \in \mathcal{F}^{p}: f^{(k)}(0)=0 \text { for } 0 \leq k \leq N\right\}
$$

are invariant subspaces for $V$.

Let us now prove that these are all the invariant subspaces of $V$. For $p=2$ this follows directly from a result of Nikol'skiu (see [15, 16]) about invariant subspaces of weighted shifts. Notice that, with respect to the standard orthonormal basis $e_{n}(z)=(n !)^{-1 / 2} z^{n}, n \geq 0$, of $\mathcal{F}^{2}$, the operator $V$ is a weighted forward shift. More precisely,

$$
V e_{n}=\frac{1}{(n+1)^{1 / 2}} e_{n+1}, \quad n \geq 0 .
$$

If the weight sequence of a weighted forward shift belongs to some $l^{s}$ with $s<\infty$, then Nikol'skil's theorem implies that its only invariant subspaces are the trivial ones, i.e.

$$
\mathcal{N}_{k}=\overline{\operatorname{Span}\left\{e_{n}: n \geq k\right\}}, \quad k \geq 0 .
$$

This is certainly the case for our operator; therefore the assertion follows.

We are now going to show that the result for $p \neq 2$ can be deduced via the case $p=2$. Assume first that $0<p<2$ and let $\mathcal{M}$ be an invariant subspace for $V: \mathcal{F}^{p} \rightarrow \mathcal{F}^{p}$. From the inclusion $\mathcal{F}^{p} \subset \mathcal{F}^{2}$ we deduce that $\overline{\mathcal{M}}^{\mathcal{F}^{2}}$ is an invariant subspace of $V: \mathcal{F}^{2} \rightarrow \mathcal{F}^{2}$ and hence

$$
\overline{\mathcal{M}}^{\mathcal{F}^{2}}=A_{N}^{2},
$$

for some $N \geq 0$. In particular, this implies that $\mathcal{M} \subseteq A_{N}^{p}$. We let $f \in A_{N}^{2}$. Then, by the above, there exists a sequence $\left(f_{n}\right) \subset \mathcal{M}$ such that $f_{n} \stackrel{\mathcal{F}^{2}}{\longrightarrow} f$ as $n \rightarrow \infty$. Now choose $M$ large enough such that $x_{M}<p$, where $\left(x_{n}\right)$ is the sequence defined in Remark 2 with $x_{0}=2$. Therefore $V^{M}: \mathcal{F}^{2} \rightarrow \mathcal{F}^{p}$ is bounded and

$$
V^{M} f_{n} \stackrel{\mathcal{F}^{p}}{\longrightarrow} V^{M} f
$$

By the invariance of $\mathcal{M}$ we get $V^{M} f_{n} \in \mathcal{M}$, and hence $V^{M} f \in \mathcal{M}$ for all $f \in A_{N}^{2}$. This last fact guarantees that the monomials $x^{k}, k \geq M+N+1$, belong to $\mathcal{M}$, and then we have

$$
A_{N+M}^{p} \subseteq \mathcal{M} \subseteq A_{N}^{p}
$$

If $\mathcal{M} \neq A_{N+M}^{p}$, let $k$ be the smallest nonnegative integer such that there exists $f \in \mathcal{M}$ with $f^{(N+1+k)}(0) \neq 0$ (clearly $\left.0 \leq k<M\right)$. But, for this particular $f$, we have $V^{M-k-1} f \in \mathcal{M}$, which in view of the first inclusion in (12) implies $z^{N+M} \in \mathcal{M}$, and therefore $A_{N+M-1} \subseteq M$. We repeat this procedure until we 
obtain $A_{N+k}^{p} \subseteq \mathcal{M}$, and then the choice of $k$ forces $\mathcal{M}=A_{N+k}^{p}$, so that the proof is done for $0<p<2$.

It remains to prove the assertion for $p>2$. Suppose $\mathcal{M}$ is an invariant subspace for $V: \mathcal{F}^{p} \rightarrow \mathcal{F}^{p}$. It follows by Theorem 1 that $V: \mathcal{F}^{p} \rightarrow \mathcal{F}^{2}$ is bounded. Then $\overline{V \mathcal{M}}^{\mathcal{F}^{2}}$ is an invariant subspace for $V: \mathcal{F}^{2} \rightarrow \mathcal{F}^{2}$, and by Nikol'skiı̌'s theorem,

$$
\overline{V \mathcal{M}}^{\mathcal{F}^{2}}=A_{N}^{2} \text {, }
$$

for some $N \geq 0$. From the above relation we obtain $\mathcal{M} \subseteq A_{N-1}^{p}$. On the other hand, since $\mathcal{F}^{2} \subset \mathcal{F}^{p}$ we have

$$
A_{N}^{2}=\overline{V \mathcal{M}}^{\mathcal{F}^{2}} \subset \overline{V \mathcal{M}}^{\mathcal{F}^{p}} \subseteq \mathcal{M}
$$

and then the monomials $z^{k}, k \geq N+1$ belong to $\mathcal{M}$. Hence $A_{N}^{p} \subseteq \mathcal{M} \subseteq A_{N-1}^{p}$. As in the case $0<p<2$, it now follows that either $\mathcal{M}=A_{N}^{p}$ or $\mathcal{M}=A_{N-1}^{p}$, which completes the proof.

\section{ACKNOWLEDGEMENTS}

While preparing this paper for submission, the author learned that results similar to those in Section 3, in the setting of several variables, were obtained recently (and independently) by Z. J. Hu [12. The author would also like to express her gratitude to the anonymous referee for constructive suggestions.

\section{REFERENCES}

[1] A. Aleman, A class of integral operators on spaces of analytic functions, Topics in complex analysis and operator theory, 3-30, Univ. Malaga, Malaga, 2007. MR2394654(2009m:47081)

[2] A. Aleman and J. A. Cima, An integral operator on $H^{p}$ and Hardy's inequality, J. Anal. Math. 85 (2001), 157-176. MR.1869606 (2002k:30068)

[3] A. Aleman and O. Constantin, Spectra of integration operators on weighted Bergman spaces, J. Anal. Math. 109 (2009), 199-231. MR2585394(2011f:47054)

[4] A. Aleman and B. Korenblum, Volterra invariant subspaces of $H^{p}$, Bull. Sci. Math. 132 (2008), 510-528. MR2445578 (2009h:47014)

[5] A. Aleman and A. G. Siskakis, Integration operators on Bergman spaces, Indiana Univ. Math. J. 46 (1997), 337-356. MR.1481594 (99b:47039)

[6] A. Aleman and A. G. Siskakis, An integral operator on $H^{p}$, Complex Variables Theory Appl. 28 (1995), 149-158. MR1700079 (2000d:47050)

[7] H.-R. Cho and K. Zhu, Fock-Sobolev spaces and their Carleson measures, preprint available at http://www.albany.edu/ kzhu/cho-zhu2.pdf.

[8] O. Constantin, Carleson embeddings and some classes of operators on weighted Bergman spaces, J. Math. Anal. Appl. 365 (2010), 668-682. MR2587070 (2011c:46051)

[9] P. Galanopoulos, D. Girela and J. A. Pelaez, Multipliers and integration operators on Dirichlet spaces, Trans. Amer. Math. Soc. 363 (2011), 1855-1886. MR2746668 (2011m:31013)

[10] D. Girela and J.A. Pelaez, Carleson measures, multipliers and integration operators for spaces of Dirichlet type, J. Funct. Anal. 241 (2006), 334-358. MR2264253 (2007m:46034)

[11] I. C. Gohberg and M. G. Krein, Introduction to the theory of linear nonselfadjoint operators. Translations of Mathematical Monographs, Amer. Math. Soc., Providence, R.I., 1969. MR0246142(39:7447)

[12] Z. J. Hu, Equivalent norms on Fock spaces with some application to extended Cesaro operators, preprint (May 2011). To appear in Proc. Amer. Math. Soc.

[13] S. Janson, J. Peetre and R. Rochberg, Hankel forms and the Fock space, Rev. Mat. Iberoamericana 3 (1987), 61-138. MR 1008445 (91a:47029)

[14] D. Luecking, Embedding theorems for spaces of analytic functions via Khinchine's inequality, Michigan Math. J. 40 (1993), 333-358. MR1226835 (94e:46046)

[15] N. K. Nikol'skiŭ, Invariant subspaces of weighted shift operators, Mat. Sb. 74 (1967), 172-190. MR0229081 (37:4659) 
[16] N. K. Nikol'skiı̌, Unicellularity and non-unicellularity of weighted shift operators, Dokl. Akad. Nauk SSSR 172 (1967), 287-290. MR0217639 (36:728)

[17] J. Pau and J. A. Pelaez, Embedding theorems and integration operators on Bergman spaces with rapidly decreasing weights, J. Funct. Anal. 259 (2010), 2727-2756 MR2679024 (2011j:46039)

[18] Ch. Pommerenke, Schlichte Funktionen und analytische Funktionen von beschränkter mittlerer Oszillation, Comment. Math. Helv. 52 (1977), 591-602. MR0454017 (56:12268)

[19] J. Rättyä, Integration operator acting on Hardy and weighted Bergman spaces, Bull. Austral. Math. Soc. 75 (2007), 431-446. MR2331020 (2008f:47056)

[20] A. G. Siskakis, Volterra operators on spaces of analytic functions - a survey, Proceedings of the First Advanced Course in Operator Theory and Complex Analysis, 51-68, Univ. Sevilla Secr. Publ., Seville, 2006. MR2290748 (2007k:47052)

[21] R. Wallstén, The $S^{p}$-criterion for Hankel forms on the Fock space, $0<p<1$, Math. Scand. 64 (1989), 123-132. MR 1036432(91c:47059)

Faculty of Mathematics, University of Vienna, Norbergstr. 15, 1090 Vienna, Austria

E-mail address: olivia.constantin@univie.ac.at 